\title{
Black Hole in the Nucleus of Active Galaxies
}

While the energy sources of the most active systems in the sky (e.g. quasars, radio-galaxies and active galactic nuclei) are still far from understood, many models invoke a gravitational source of energy and in particular, a massive black hole. If a supermassive object such as a black hole lies at the centre of an active system, the surrounding matter will fall in towards the centre and a part of the kinetic energy of the infalling gas will be radiated. If all the gravitational energy were transformed into radiation, the energy output would correspond to $10^{8}$ solar masses (1 solar mass $=1 \mathrm{M}_{\Theta}=2 \times 10^{33} \mathrm{~g}$ ).

The presence of a massive core would have observable effects on the nucleus. The large concentration of stars bound to it will create a central bump in the luminosity profile, which can be observed using photometric techniques, and the large gravitational potential will increase the velocity dispersion of the surrounding stars, which can be measured from the broadening of the absorption line spectra.

The peculiar elliptic galaxy M 87 which lies in the centre of the Virgo cluster of galaxies appeared to be a particularly promising candidate for sheltering a supermassive core. It is relatively close $\left(15 \mathrm{MPc}=4.5 \times 10^{20}\right.$ $\mathrm{m})$, it is known to be an active radio source with a compact component (1.3 arc $\mathrm{ms}$ ), and there is optical evidence of ejection of mass from the nucleus in the form of a jet. The total anomalous radiation output from the galaxy is $2 \times 10^{42} \mathrm{erg} \mathrm{s}^{-1}$ which places it among the weakest of the radio sources associated with supergiant elliptical galaxies.

The search for a supermassive object was undertaken by two teams of astronomers *, most of whom came from the California Institute of Technology. One made photometric studies with the 200 in and 60 in telescopes in Palomar, the second made spectrometric measurements with the $4 \mathrm{~m}$ telescope at Kitt Peak National Observatory in Arizona.

For the photometric studies two different electronic imaging systems were used: an SIT (silicon intensified target) television-camera tube, and a CCD (charge coupled device) camera which is particulary suitable for two dimensional photometry of faint objects since it has a wide dynamic range and no image distortion. It has, moreover, a high spatial resolution: $0.25^{\prime \prime}$ at the 200 in prime focus. The accurate luminosity map shows the presence of a tiny bright spot which lies within 0.02 arc $s$ of the centre of the galaxy, and a luminosity excess, compared to the luminosity profile of normal elliptical galaxies, which extends up to 20 arc $s$ from the centre. The luminosity profile could be explained by assuming that the centre of the galaxy had an excess of massive stars, but this kind of model fails to explain the observed velocity dispersion, predicting instead a constant velocity dispersion across the core.

The velocity study was done using a two dimensional photon counting system designed by $A$. Boksenberg of University College, London. Spectra taken at different distances from the centre along the main axis of the galaxy, showed that the dispersion increases sharply from $230 \mathrm{~km} / \mathrm{s}$ in the external part to $290 \mathrm{~km} / \mathrm{s}$ at 10 arc s, and $350 \mathrm{~km} / \mathrm{s}$ at 1.5 arc $\mathrm{s}$ from the centre. For comparison, a similar measurement made on the normal elliptical galaxy NCC 3379 shows only a very moderate increase $(190 \mathrm{~km} / \mathrm{s}$ in the external part to $220 \mathrm{~km} / \mathrm{s}$ in the central part). The velocity dispersion data are consistent with a very compact object which appears as a point source to its surroundings.

\section{Nominal D-T Ignition Temperature Exceeded}

The 7th International Conference on Plasma Physics and Controlled Nuclear Fusion, organized by the International Atomic Energy Agency, was held at Innsbruck from 23 to $30 \mathrm{Au}$ gust, 1978. It was attended by about 500 scientists from all over the world, two thirds of whom were European.

The most important single result reported at the Conference was in the field of magnetic plasma confinement which continues to receive most attention. This was the attainment of an ion temperature of $5.5 \mathrm{keV}$ in the Princeton Large Tokamak by injecting neutral particle beams carrying $2 \mathrm{MW}$ for $100 \mathrm{~ms}$. Such a temperature is higher than that required to make fusion- $\alpha$-particle selfheating of a deuterium-tritium plasma larger than its bremsstrahlung loss: in other words the nominal ignition temperature of a DT plasma has been exceeded, and the range of thermonuclear fusion ion temperatures enter-
Combining their results, the teams concluded that within 1.5 arc $s$ (110 $\mathrm{Pc}$ ) of its centre, $M 87$ has an excess mass of $5 \times 10^{9} \mathrm{M}_{\Theta}$. Moreover this mass is very dim since the luminosity increase is 10 times smaller than it would be if the mass were in the form of normal stars.

This all points to the existence of a massive black hole in the centre of M 87. It should be emphasized that it is not the only way in which the data can be explained, but in many respects it is the most attractive. Whatever the actual nature of this supermassive heart of $M 87$, its discovery will have important consequences for our understanding of very active objects in the sky. Unfortunately, present observations are at the limit of what is possible from ground-based observations and significant improvements in the data on M 87 or other similar galaxies wili not be achieved until the launching of the Space Telescope.

(a) YOUNG, P.J., WESTPHAL, J.A., KRISTIAN, J., WILSON, C.P. and LANDAUER, F.P. Astrophysical Journal 221 (1978) 721

(b) SARGENT, W.L.W., YOUNG, P.J., BOKSENBERG, A., SHORTRIDGE, K., LYNDS, C.R. and HARTWICK, F.D.A. Astrophysical Journal 221 (1978) 731.

L. Vigroux (C.E.N. Saclay) ed. Even more important is that no deleterious effects on plasma confinement were observed, in contrast to theoretical expectations which forecast new types of instabilities as collision effects in the plasma decreased in strength.

The ion heating experiment was done at relatively low plasma densities (about $10^{13}$ particles $/ \mathrm{cm}^{3}$ ) which implies that the electron temperature of $2 \mathrm{keV}$, reached by ohmic heating, was increased by only $50 \%$ to about $3 \mathrm{keV}$, and the overall energy confinement time, determined by electron heat losses, was relatively short, namely $30 \mathrm{~ms}$. Consequently the product of density and confinement time is more than two orders of magnitude below that necessary for a DT plasma to be self-sustained by fusion heating. The maximum value of this product attained in tokamaks has been reported to have reached $3 \times 10^{13} \mathrm{~cm}^{-3} \mathrm{~s}$ in ALCATOR at MIT in discharges 
having peak densities of more that $10^{15}$ particles $/ \mathrm{cm}^{3}$ and temperatures close to $1 \mathrm{keV}$. The scaling of confinement, proposed on the basis of earlier results in this machine, was confirmed by the Frascati Tokamak which has come into operation during the past year, showing in particular that confinement improves quadratically with the radius of the plasma column. The machine extends the parameter range of plasma currents in high-field machines to $600 \mathrm{kA}$.

The intense work aimed at understanding in more detail, controlling discharges and improving plasma quality has continued. Focusing points here are the magnetohydrodynamic fluctuations, the disruption phenomenon, operation close to the magnetohydrodynamic stability limit, the energy and particle transport mechanisms, and the problems of plasma contamination by impurities and of impurity behaviour in the discharge; arguments where all running tokamaks have contributed new information. Using carbon or steel rather than heavy metal limiters, as well as gettering, has reduced the impurity content of hot low-density discharges appreciably, and the effectiveness of divertors in keeping the plasma clean has been further assessed.

As far as radiofrequency heating is concerned, interesting new results have been reported, in particular for the lower-hybrid frequency range. (EN 9 (1978) $1 / 2$ p. 9). Work done by the JFT-2 group at Tokai and by the PETULA and WEGA teams at Grenoble has shown that the coupling of power to the plasma is good (about 80 to $90 \%$ ) and bacomes even better when the power is increased. At low power levels at least, the heating efficiency is almost as high as for neutral injection (ca. $1 \mathrm{eV}$ ion temperature increase per $\mathrm{kW}$ injected power at plasma densities of about $10^{13}$ particles $/ \mathrm{cm}^{3}$ ) and the plasma confinement is not impaired by the presence of the waves.

The parameter range of Stellerator discharges has been further extended. In Wendelstein VII A at Garching, a density of $10^{14}$ particles $/ \mathrm{cm}^{3}$ has been reached in the discharges. This device as well as $\mathrm{L}-2$ at the Lebedev Institute (Moscow) and CLEO at Culham have further provided new insight into the physics of toroidally confined plasmas.

Work on high- $\beta$ toroidal confinement systems continues on a limited scale. One of the main points here is the investigation of the self-reversal me-

chanism in reversed-field pinches, mainly done in HBTX $I$ at Culham and ETA-BETA I at Padova.

Detailed understanding of the underlying physics of magnetic mirror confinement continues to improve. In 2X II B at Livermore it was shown that plasma confinement definitely becomes better with increasing values of the ratio of plasma radius to the ion Larmor radius. In the attempts to generate close field lines by field reversal, a reduction of the magnetic field on the mirror axis of $90 \%$ has been attained. The tandem mirror concept is intensely studied theoretically and experiments are being prepared. First experimental work on the concept performed on the device Gamma 6 at the University of Tsukuba has been reported and shows clearly an improvement of plasma confinement.

\section{Seventy-fifth Anniversary of the Spanish Royal Society of Physics and Chemistry}

On 2 October, the Spanish Royal Society of Physics and Chemistry celebrated its 75 th anniversary. European physics was represented by the President of EPS, Antonino Zichichi who on behalf of the Society conveyed to the President of the Spanish society, Professor J.M. Gamboa Loyarte his warmest greetings. At the commemoration ceremony, held in the presence of is Majesty the King, members of the Government and an international gathering of scientists, Zichichi reminded his audience of the uniqueness of EPS wherein all European countries, regardless of political structure, were represented in one organization that comprised over 3000 Individual Ordinary Members and 28 National Societies and Academies representing some 35000 physicists from all over Europe - East, West, North and South.

The basis for this new unity of geographical Europe, lay in what be called the new culture, viz. the scientific culture. He continued: "As you know, the greatest inventions of human intellect are just three: Language (invented a few hundred thousand years ago), Logic (three thousand years) and Science (just 350 years old). The culture of man today, so-called modern man, is based on and dominated by language. Logic and Science have very little influence on the culture of our time. This is why millions of people can be influenced by just a slogan. A few words, which are an insult to
In the field of inertial confinement a strong effort is made to develop more powerful laser and particle beams for plasma compression. In parallel, the work on the physics of compression and heating of pellets by beams continues.

Conceptual designs of fusion reactors were reported mainly for confinement schemes other than tokamaks, thus filling an important gap, while for tokamaks, attention is being given more and more to economic questions. Furthermore, fusion systems which aim at producing hydrogen gas or at breeding fissile material ("fusionfission hybrid reactors") rather than generating electricity are now under discussion.

F. Engelmann

(Chairman Plasma Physics Division) the human intellect, are enough to create tragedies world wide. The root of the divisions among people of our planet is the fact that logic and science are still not part of modern human culture. As scientists it is our duty to identify all points where unity - not division can be created among people of different religions, classical cultures and political organizations. These points exist in science, and this is why the European Physical Society exists. Your 75 th anniversary coincides with the 10th anniversary of EPS, of which you are an important component. My presence here is proof of how much we want to strengthen the links between the scientists of Spain and the rest of Europe...

It is one of the most important duties of EPS to help all physicists and national societies of Europe to play an active role in the development of our science. It is in this spirit that EPS honours the 75th Anniversary of the Royal Society of Physics and Chemistry of Spain, by awarding to the Society, five scholarships that will allow five Spanish physicists to follow the International Courses at the Ettore Majorana International Centre for Scientific Culture in $1979 . .$.

This month marks ten years of life in the unity of all European physicists and the turning point in a new intensive cultural collaboration among all the physicists of Europe. 